\title{
Abordagem didática para a popularização da Internet das Coisas na Educação Básica
}

\author{
André Pinto Moreira ${ }^{1}$, Gabriel Jaime Alves ${ }^{1}$, Carlos Roberto Beleti Junior ${ }^{1}$, Daniela \\ Eloise Flôr ${ }^{2}$, Linnyer Beatrys Ruiz Aylon ${ }^{3}$ \\ ${ }^{1}$ Universidade Federal do Paraná (UFPR) \\ Campus Jandaia do Sul - PR - Brasil \\ ${ }^{2}$ Instituto Federal do Paraná (IFPR) \\ Campus Paranavaí - PR - Brasil \\ ${ }^{3}$ Universidade Estadual de Maringá (UEM) \\ Maringá - PR - Brasil \\ \{moreira.andre.p,ga.jaimealves\}@gmail.com, carlosbeleti@ufpr.br, \\ daniela.flor@ifpr.edu.br, lbruiz@uem.br
}

Abstract. The Internet of Things aims to interconnect objects with each other and with people, through data transmission technologies and various sensors and actuators. In Education, initiatives have been promoted to introduce the Internet of Things in schools, to demonstrate the possibilities of this new paradigm as an aid tool in interdisciplinary teaching activities. Thus, this work aims to demonstrate the results of the application of a didactic-pedagogical approach composed of one Didactic Sequence for the area of Physics for high school students, using a prototype of a smart home built with based on the IoT paradigm.

Resumo. A Internet das Coisas tem como objetivo interconectar objetos entre si e com pessoas, por meio de tecnologias de transmissão de dados e diversos sensores e atuadores. Na Educação, tem sido promovidas iniciativas de introdução de Internet das Coisas nas escolas, visando demonstrar as possibilidades desse novo paradigma como ferramenta de auxílio nas atividades de ensino interdisciplinar. Com o exposto, este trabalho tem o objetivo de demonstrar os resultados da aplicação de uma abordagem didático-pedagógica composta por uma Sequência Didática para a área da Física para alunas e alunos do Ensino Médio, utilizando um protótipo de uma casa inteligente construída com base no paradigma de IoT.

\section{Introdução}

Com a evolução das Tecnologias Digitais de Informação e Comunicação (TDIC), e das Redes de Sensores Sem Fio (RSSF), o paradigma da Internet das Coisas vem se consolidando nos últimos anos e já é uma realidade no cotidiano das pessoas. Segundo [Araújo e Valentim, 2019] "Internet das Coisas (IoT - do inglês Internet of Things) é um paradigma tecnológico que realiza a interconexão entre os mundos físico e digital, criando um 'meio informacional' que aumenta a percepção das pessoas sobre o cenário ao seu redor".

A IoT é um tópico de emergência para o desenvolvimento técnico, social e econômico. A crescente popularização desta área nos principais segmentos da sociedade, e a combinação de conectividade com a internet em produtos de consumo, bens duráveis, carros 
e caminhões, sensores e objetos, juntamente com poderosas ferramentas analíticas de dados, está transformando o modo como as pessoas vivem [Kharchenko et al., 2017, p. 1].

Atualmente a IoT está presente em ambientes escolares, promovendo mudanças e quebras de paradigmas no modelo educacional tradicional. Segundo [Savov et al., 2017], "pesquisadores concordam que a sala de aula deve integrar tecnologias inteligentes e uma pedagogia inovadora".

Com exposto, este trabalho tem o objetivo de apresentar os resultados da aplicação de uma abordagem didático-pedagógica de ensino de conceitos de Física que utiliza a IoT por meio de Sequência Didática (SD), ferramentas tecnológicas e um protótipo de uma casa inteligente. Tal abordagem foi aplicada à duas turmas do Ensino Médio de um colégio público da cidade de Jandaia do Sul.

\section{Trabalhos relacionados}

[Tavares et al., 2018], apresentam uma experiência que integra o método BYOD (Bring Your Own Device) ao cenário da IoT que estimula o aluno para utilizar seus próprios dispositivos dentro do ambiente de aprendizado. Nessa atividade os alunos tinham como ferramenta smartphones e tablets, que segundo os autores, seriam tecnologias IoT inteligentes. Por meio dos dispositivos, os alunos acessaram documentos de textos e vídeos para entender o tema IoT, descrevendo os conceitos aprendidos, em um documento compartilhado em tempo real.

Em [Gingl et al., 2019], os autores apresentam o EDAQuino, um dispositivo desenvolvido com objetivo de facilitar o ensino de conceitos STEM (Science, Technology, Engineering e Mathematics) por meio do sensoriamento do ambiente. Esses dispositivos que são desenvolvidos para serem acoplados em uma placa Arduino são conhecidos como shields. Cada dispositivo vem com um sensor fotodetector infravermelho integrado, e outros sensores podem ser facilmente acoplados, como termostato, acelerômetro, entre outros.

[Silveira e Girardi, 2016] descrevem a construção e o funcionamento de um kit experimental, de baixo custo para demonstrar o efeito fotoelétrico. Foi utilizada a plataforma Arduino para o controle e a comunicação com um computador, um sensor pico-amperímetro para fazer a leitura da corrente elétrica, um conjunto de lâmpadas montadas com LEDs comuns integrados em uma caixa metálica, uma lâmpada UV (lâmpada de mercúrio de cátodo frio), grade e placa de zinco e uma fonte de alta tensão.

[Moreira, Romeu et al., 2018], apresentam uma revisão da literatura com iniciativas que utilizam o Arduino no ensino de Física. Foi realizado um levantamento com base em 20 artigos selecionados, nos quais apenas 3 foram realmente testados em ambientes didáticos, e apresentaram resultados satisfatório quanto ao uso de tecnologia. Os demais trazem propostas de experimentos de Física utilizando o Arduino, voltados para o ensino médio. As áreas da Física mais abordadas nos artigos, foram: 40\% termologia, 25\% mecânica, 15\% Física Moderna, $15 \%$ eletromagnetismo e $5 \%$ ondas.

\section{Casa Inteligente: MannaHome}

O protótipo de casa inteligente, denominado MannaHome, foi desenvolvido por integrantes do projeto de pesquisa MannaAcademy ${ }^{1}$. Esse projeto, tem como objetivo estabelecer uma rede de estímulo à participação e à formação de meninas e mulheres nas áreas de STEM para

\footnotetext{
${ }^{1}$ Página do grupo Manna: https://pt-br.facebook.com/mannateam
} 
as carreiras de Computação, Microeletrônica e Engenharias. A Figura 1 - a), apresenta alguns sensores e atuadores que foram utilizados na MannaHome. Dentre esses sensores, alguns foram escolhidos para serem utilizados nos experimentos realizados em sala.

A Figura 1 - b) apresenta a MannaHome com todas as placas e sensores acoplados. As placas utilizadas no protótipo foram: o Arduino MEGA 2560 R3, ESP8266 e ESP32. O Arduino fica responsável por controlar a parte da garagem da MannaHome, que conta com um sistema RFID, um kit infravermelho e um servo motor que aciona o portão, e as duas placas são responsáveis por toda a transmissão dos dados captados para a internet.
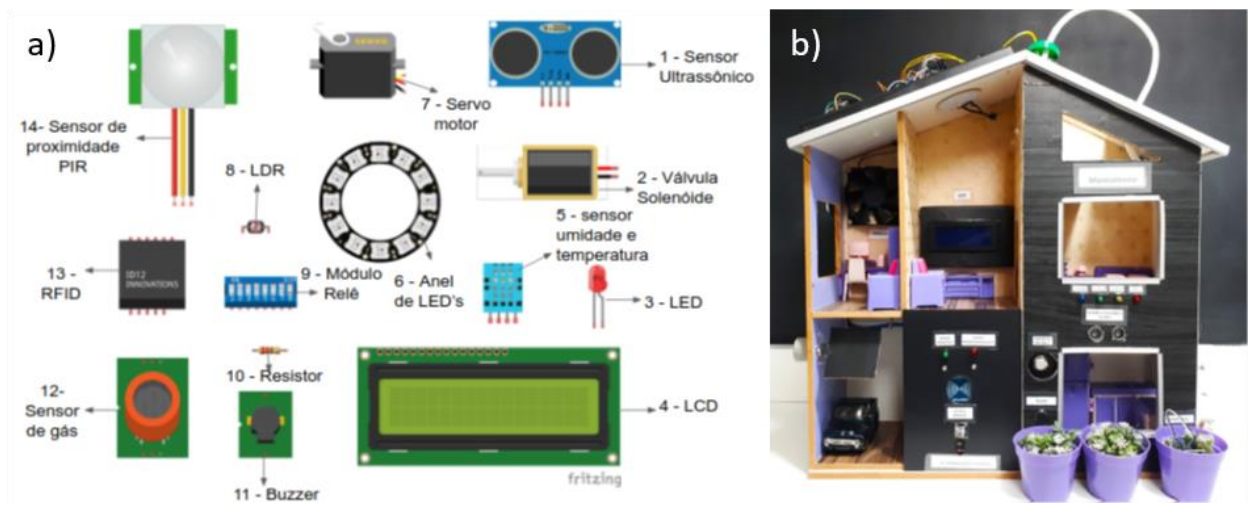

Figura 1 - a) Sensores e atuadores utilizados na MannaHome; b) MannaHome.

Além das placas, componentes e sensores, o protótipo contou com a implementação que foi realizada em linguagem $\mathrm{C} / \mathrm{C}++$ com duas funções principais, sendo elas: setup() e $\operatorname{loop}()$. A função setup() é acionada uma única vez, assim que o programa é executado. A função loop() pode ser executada repetidamente. Todas as outras funções ou trechos de código são chamadas a partir dessas duas funções principais [Barai, Biswas e Sau, 2017].

Os dados captados pelos sensores da MannaHome são transmitidos a uma plataforma na nuvem chamada CloudMQTT ${ }^{2}$, que funciona como um broker MQTT ${ }^{3}$ rodando na nuvem podendo ser configurado via web. Essa aplicação é um cliente MQTT, que permite a visualização dos dados recebidos pelo broker, que neste caso, está hospedado no CloudMQTT. O aplicativo MQTT Dash foi utilizado para a visualização destes dados no smartphone. Ele está disponível para download na Play Store para smartphones que utilizem o sistema operacional Android.

\section{Resultados e discussão}

A abordagem didático-pedagógica foi desenvolvida com intuito de disseminar a IoT para os jovens em idade escolar. A forma encontrada para isso, foi unir conceitos de Física que os alunos aprendem nas escolas com suas aplicações na prática, que podem ser abordados, por exemplo, nos componentes (sensores/atuadores) da MannaHome.

A Sequência Didática foi aplicada no laboratório de práticas pedagógicas, do curso de Licenciatura em Computação da Universidade Federal do Paraná, Campus Avançado em Jandaia do Sul. O público alvo foram alunos do $2^{\circ}$ ano do Ensino Médio do Colégio Estadual Rui Barbosa, localizado no município de Jandaia do Sul. A sequência teve duração aproximada de 70 minutos, tempo disponibilizado pelo colégio no dia da aplicação, ou seja, foi realizada em apenas uma aula.

\footnotetext{
2 Disponível em: https://www.cloudmqtt.com/

${ }^{3}$ Disponível em: https://www.ibm.com/developerworks/br/library/iot-mqtt-why-good-for-iot/index.html
} 
Inicialmente, foi apresentada a proposta da aula aos alunos. Em seguida, foi realizado um quiz, com o auxílio de um sistema de resposta à audiência (SRA), através dos clickers, com intuito de verificar o conhecimento prévio dos alunos. Esse quiz foi reaplicado ao fim da aula, que ocorreu no mesmo dia, para tentar registrar indicativos de aprendizado. Nesse primeiro momento, as respostas corretas não foram disponibilizadas aos alunos.

Em seguida, foi realizada uma breve descrição a respeito da IoT, seguido de questionamentos como, por exemplo: "O que uma geladeira precisa ter, para se tornar uma geladeira inteligente?"; "Como seria uma cidade inteligente?"; "Na sua casa há dispositivos IoT ?". Ao término desse momento, foram apresentados alguns exemplos de aplicações IoT, que já estão disponíveis para serem utilizadas pela população, como: Carros semiautônomos, lixeira inteligente, assistentes pessoais, chuveiros inteligentes. Foram apresentados os microcontroladores, os sensores e atuadores, de forma individual, e como eles integravam o sistema da casa, a MannaHome.

Mostrou-se como é possível controlar os dispositivos da casa por meio de um computador com acesso à internet. Com essas aplicações, foi possível demonstrar o acionamento dos componentes da casa, como as luzes e o ventilador, e fazer a leitura dos dados coletados pelos sensores. A saber: umidade relativa do ar, temperatura ambiente, nível de gás na atmosfera, umidade do solo, sensor de chuva, sensor de presença, sensor de proximidade e o nível de luminosidade ambiente. Para finalizar a aula, foi aplicado novamente o quiz, desta vez mostrando um feedback visual com a porcentagem de acertos e erros de cada pergunta, após serem respondidas, conforme visualizado na Figura 2 - a) e b).

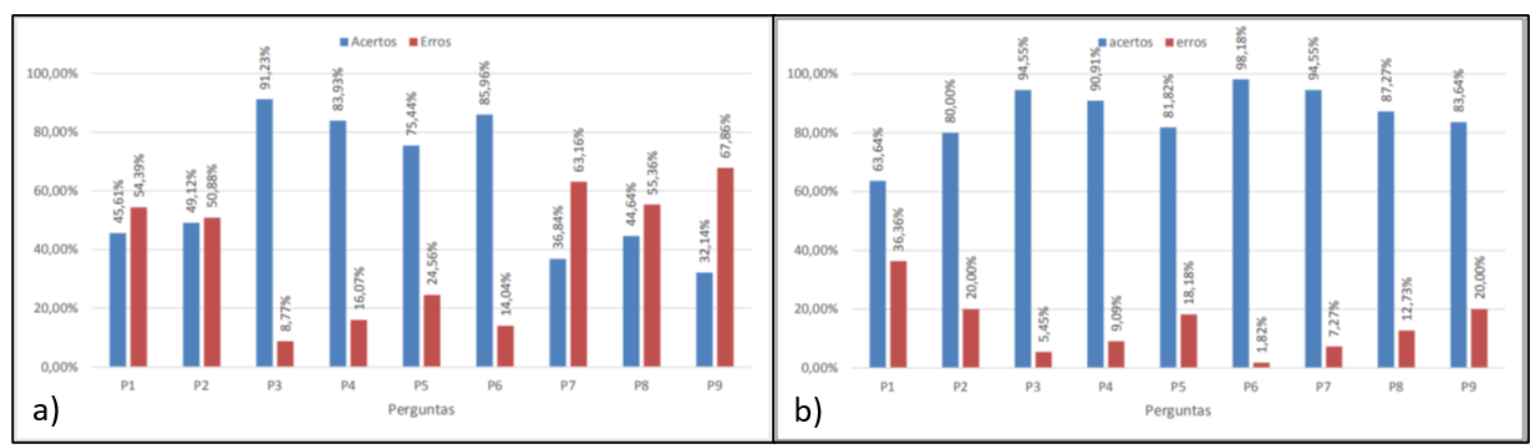

Figura 2 - a) Resultados do quiz pré-teste; b) Resultados do quiz pós-teste.

$\mathrm{Na}$ aplicação dos formulários online do Google, os alunos estavam dispostos em grupos para debaterem as perguntas e formularem as respostas, estimulando o trabalho em equipe. Apesar da maioria relatar que não conhecia o termo Internet das Coisas ou IoT, grande parte das questões relacionadas a esse conceito foram acertadas. A questão que apresentou o maior ganho percentual, em relação a quantidade de respostas corretas entre o pré-teste e o pós-teste, foi a P7, com um ganho, em média, de 57,71 pontos percentuais.

Com base na Figura 2 - a) e b), os resultados se mostraram positivos na comparação da média entre os erros e acertos. Como observado, houve um aumento em mais de 25 pontos percentuais, tendo uma melhoria de mais de $40 \%$ nas respostas corretas, indicando uma tendência de aprendizado por parte dos alunos.

\section{Considerações Finais}

Após realizada a análise dos dados apresentados neste trabalho, observou-se como os alunos se interessam por atividades que envolvam o uso de novas tecnologias da qual não estão 
acostumados a terem contato em um ambiente de ensino tradicional, e como essas tecnologias podem potencializar o ensino e o aprendizado de forma mais dinâmica.

Os alunos participaram de um pré-teste e um pós-teste, por meio de um sistema de resposta à audiência que realiza a coleta dos dados, com intuito de verificar o conhecimento anterior e o conhecimento adquirido durante a aplicação da Sequência Didática. De modo geral, apesar da realização das atividades ter ocorrido no mesmo dia e com duração de apenas 70 minutos, os alunos tiveram uma evolução quanto ao número de respostas corretas, indicando uma possível compreensão dos conceitos abordados. $\mathrm{O}$ ponto positivo da utilização de SRAs é o fato da dinâmica com os clickers ser mais atrativa para o aluno, tendo um feedback visual imediato, o que coopera no engajamento dos alunos.

Apesar dos resultados demonstrarem uma tendência de melhoria no aprendizado, não se tem a pretensão de substituir as ações didáticas do professor em um ensino tradicional, por esse tipo de abordagem, mas busca-se complementá-las, de uma forma mais dinâmica e que seja mais atrativa para os alunos. É importante refletir ainda sobre as estruturas das escolas, pois grande parte delas não possuem recursos para a realização de tais atividades, pois carecem de acesso à internet de qualidade, além da falta de equipamentos de hardware.

\section{Agradecimentos}

Este trabalho tem o apoio do CNPq (Processo/MCTIC 442433/2018 e 426119/2016), bem como das demais instituições de ensino envolvidas.

\section{Referências}

Araújo, C. A. Á.; Valentim, M. L. P. A Ciência da Informação no Brasil: mapeamento da pesquisa e cenário institucional. Bibliotecas. Anales de Investigación, v. 15, n. 2, p. 232 259, 2019.

Barai, S.; Biswas, D.; Sau, B. Estimate distance measurement using NodeMCU ESP8266 based on RSSI technique. In: IEEE. 2017 IEEE Conference on Antenna Measurements \& Applications (CAMA). [S.1.: s.n.], 2017. p. 170-173.

Gingl, Z. et al. Universal Arduino-based experimenting system to support teaching of natural sciences. arXiv preprint arXiv:1901.03810, 2019.

Kharchenko, V. et al. Emerging curriculum for industry and human applications in Internet of Things. In: IEEE. 2017 9th IEEE International Conference on Intelligent Data Acquisition and Advanced Computing Systems: Technology and Applications (IDAACS). [S.1.: s.n.], 2017. v. 2, p. 918-922.

Moreira, M.; Romeu, M. C. et al. Contribuições do Arduino no Ensino de Física: uma revisão sistemática de publicações na área do ensino. Caderno Brasileiro de Ensino de Física, Universidade Federal de Santa Catarina, v. 35, n. 3, p. 721-745, 2018.

Savov, T. et al. Contemporary technology support for education. In: CBU International Conference Proceedings. [S.1.: s.n.], 2017. v. 5, p. 802-806.

Silveira, S.; Girardi, M. Desenvolvimento de um kit experimental com Arduino para o ensino de física moderna no ensino médio. Revista Brasileira de Ensino de Física, Universidade Federal de Santa Catarina, Araranguá, SC, Brasil., v. 39, n. 4, e405-2-e405-7, 2016.

Tavares, S. et al. Internet das coisas na Educação estudo de caso e perspectivas. South American Development Society Journal, v. 4, n. 10, p. 99-112, 2018. 